\title{
Effects of pressure and heat loss on the unstable motion of cellular-flame fronts caused by intrinsic instability in hydrogen-air lean premixed flames
}

\author{
Satoshi KADOWAKI*, Thwe Thwe Aung**, Taisei FURUYAMA***, Kazumasa KAWATA***, \\ Toshiyuki KATSUMI*** and Hideaki KOBAYASHI**** \\ *Department of System Safety, Nagaoka University of Technology \\ 1603-1 Kamitomioka, Nagaoka 940-2188, Japan \\ E-mail: kadowaki@mech.nagaokaut.ac.jp \\ ${ }^{*}$ Collaborative Laboratories for Advanced Decommissioning Science, Japan Atomic Energy Agency \\ 4002 Oarai, Ibaraki 311-1393, Japan \\ ***Department of Mechanical Engineering, Nagaoka University of Technology \\ 1603-1 Kamitomioka, Nagaoka 940-2188, Japan \\ ****Institute of Fluid Science, Tohoku University \\ 2-1-1 Katahira, Aoba-ku, Sendai 980-8577, Japan
}

Received: 23 November 2020; Revised: 12 January 2021; Accepted: 28 January 2021

\begin{abstract}
Effects of pressure and heat loss on the unstable motion of cellular-flame fronts in hydrogen-air lean premixed flames were numerically investigated. We adopted the reaction mechanism for hydrogen-oxygen combustion, modeled with seventeen reversible reactions of eight reactive species and a diluent. Two-dimensional unsteady reactive flow was treated, and the compressibility, viscosity, heat conduction, molecular diffusion and heat loss were taken into account. A sufficiently small disturbance was superimposed on a planar flame to obtain the relation between the growth rate and wave number, i.e. the dispersion relation, and the linearly most unstable wavelength, i.e. the critical wavelength. As the pressure became higher, the maximum growth rate increased and the unstable range widened. These were due mainly to the decrease of flame thickness. As the heat loss became larger, the former decreased and the latter narrowed, which were due mainly to the decrease of burning velocity. To investigate the characteristics of cellular-flame fronts, a disturbance with the critical wavelength was superimposed. The superimposed disturbance developed owing to intrinsic instability, and then the cellular shape of flame fronts appeared. The burning velocity of a cellular flame normalized by that of a planar flame increased as the pressure became higher and the heat loss became larger. This indicated that the pressure and heat loss affected strongly the unstable motion of cellular-flame fronts. The burning velocity of a cellular flame increased monotonically with an increase in the space size. This was attributed to long-wavelength components of disturbances. Moreover, we estimated the fractal dimension of flame fronts through the box counting method. As the pressure and heat loss increased, the fractal dimension became larger, which denoted that the flame shape became more complicated.
\end{abstract}

Keywords : Hydrogen, Lean premixed flame, Pressure, Heat loss, Unstable motion, Cellular-flame front, Intrinsic instability, Fractal dimension

\section{Introduction}

Recently, we pay attention to emissions of carbon dioxide as greenhouse gases and nitrogen oxides as air pollutants, because most of energy is derived from the combustion of fossil fuels including coal, petroleum, natural gas, oil shales and heavy oil. Reducing these emissions to suppress global warming and air pollution, we put the spotlight on lean combustion of hydrogen-air premixtures. Hydrogen-air lean premixed flames generate no carbon dioxide and reduce the production of nitrogen oxides because of low flame temperature. To control lean hydrogen flames effectively and 
safely, it is significant to obtain the fundamental knowledge on the unstable motion of cellular-flame fronts caused by intrinsic instability.

Intrinsic instability is attributed to hydrodynamic instability and diffusive-thermal instability. The former instability caused by thermal expansion is essential to the intrinsic instability of all premixed flames, and the latter instability caused by preferential diffusion of mass versus heat affects the instability of lean hydrogen flames. Hydrodynamic and diffusive-thermal instabilities were studied through experimental, theoretical and numerical approaches, and the essential mechanism on formation of cellular-flame fronts was revealed (Williams, 1985; Clavin, 1985; Hertzberg, 1989; Sivashinsky, 1990; Kadowaki and Hasegawa, 2005). In hydrogen-air premixed flames, the formation of cellular fronts caused by intrinsic instability is observed in the experiments (Mitani and Williams, 1980; Yanez et al., 2015), and especially in spherically expanding flames (Kim et al., 2013; Okafor et al., 2014; Nakahara et al., 2015; Katsumi et al., 2017). In addition, the instability of premixed flames was numerically studied in detail (Pizza et al., 2009; Kadowaki et al., 2015; Frouzakis et al., 2015).

In the industrial application of premixed combustion of hydrogen under the high-pressure conditions, e.g. premixedtype gas turbines, flame instability is important factor in operating machinery (Ichikawa et al., 2011; Park and Lee, 2016a; Park and Lee, 2016b; Imteyaz et al., 2018). Clarifying the effects of pressure on intrinsic instability is significant to handle premixed-type combustion machinery. We have several reports regarding the combustion characteristics of hydrogen-air premixtures under the high-pressure conditions (Qin et al., 2000; Hu et al., 2009a; Gubernov et al., 2017). Based on the previous information on the effects of pressure, we investigate the characteristics of unstable motion of hydrogen flames caused by intrinsic instability. This investigation contributes to the effective and safe handling of combustion machinery under the high-pressure conditions.

The heat loss appears in most of combustion and affects the intrinsic instability of premixed flames, especially at Lewis numbers lower than unity, i.e. hydrogen-air lean premixed flames. The unstable motion of premixed flames with low Lewis numbers becomes stronger as the heat loss increases (Joulin and Clavin, 1979; Chao, 2001; Bechtold et al., 2005; Kadowaki, 2005). In hydrogen-air premixed combustion, the effects of heat loss on the flame characteristics were studied experimentally and numerically (Xiao et al., 2015; Yenerdag et al., 2016; Gavrikov et al., 2019). It was reported that the heat loss plays a significant role in the characteristics of hydrogen flames. Now it is necessary to further develop the research on intrinsic instability of hydrogen-air lean premixed flames with heat loss under the high-pressure conditions.

Clarifying the complexity of flame fronts quantitatively, it is appropriate to estimate the fractal dimension. To elucidate the characteristics of disturbed flame fronts, the fractal dimension was obtained by means of the box-counting method in numerical calculations of laminar and turbulent flow (Miyauchi et al., 1994; Mukaiyama et al., 2013; Battista et al., 2015). The fractal dimension occupies a valuable position in the characteristics of cellular-flame fronts caused by intrinsic instability.

In our previous numerical calculations, we handled the unsteady reactive flow, based on the detailed chemical reaction model, to clarify the role of intrinsic instability in the unstable behavior of hydrogen-air lean premixed flames in large space under the non-adiabatic conditions (Kadowaki et al., 2020). However, we did not perform calculations under the high-pressure conditions, even though the pressure plays a crucial role in combustion phenomena, nor obtain the fractal dimension. It is well-known that the burning velocity of hydrogen-air premixed flames decreases as the pressure becomes higher (Bradley et al., 2007; Hu et al., 2009b; Dayma et al., 2014), and that the flame thickness becomes thinner because of the increase of mass burning rate. The burning velocity and flame thickness, depending on the pressure, have a great influence on intrinsic instability. Thus, we have to elucidate the pressure effects on unstable motion and to estimate the fractal dimension of flame fronts in order to obtain the fundamental knowledge for controlling lean combustion of hydrogen-air premixtures.

In this investigation, we treated hydrogen-air lean premixed flames and calculated two-dimensional unsteady reactive flow, based on the compressible Navier-Stokes equations including detailed chemical reactions. The effects of pressure and heat loss on the unstable motion of cellular-flame fronts caused by intrinsic instability were investigated, and the increase of normalized burning velocity due to high pressure and large heat loss was elucidated. Moreover, we estimated the fractal dimension through the box counting method to clarify the complexity of flame fronts quantitatively. 


\section{Basic equations}

In this calculation, we adopted the reaction mechanism for hydrogen-oxygen combustion proposed by Westbrook (1982), modeled with seventeen reversible reactions of eight reactive species, i.e. $\mathrm{H}_{2}, \mathrm{O}_{2}, \mathrm{H}, \mathrm{O}, \mathrm{OH}, \mathrm{HO}_{2}, \mathrm{H}_{2} \mathrm{O}_{2}$ and $\mathrm{H}_{2} \mathrm{O}$, and $\mathrm{N}_{2}$ as a diluent. Getting the transport coefficients of pure gases, we used the formulae founded on the rigorous kinetic theory (Hirschfelder et al., 1954). Diffusion coefficient, dynamic viscosity and thermal conductivity of each species in gaseous mixtures were estimated by the mixture rules (Wilke, 1950a; Wilke, 1950b; Coffee and Heimerl, 1981).

The ideal gas equation of state was employed in the governing equations; the body force, bulk viscosity, radiation, Soret effect, Dufour effect and pressure gradient diffusion were neglected. The heat loss was assumed to obey the Newton's law of cooling, i.e. proportional to the difference in temperature between reactive gas and unburned gas, because we considered premixed flames propagating in a narrow space where the wall temperature was equal to the unburned-gas temperature. We considered two-dimensional unsteady reactive flow and took the direction tangential to the flame front as the $y$-direction, with the velocity in the positive $x$-direction.

The basic equations of two-dimensional unsteady flow, i.e. the equation of state, the equation of continuity for $i$-th species, the equation of continuity, the equation of motion in the $x$-direction, the equation of motion in the $y$-direction and the equation of energy, were written as follows:

$$
\begin{aligned}
& p=\left(\sum_{i} C_{i}\right) R T \\
& \frac{\partial}{\partial t}\left(\rho Y_{i}\right)+\frac{\partial}{\partial x}\left(\rho Y_{i} u-\rho D_{i} \frac{\partial Y_{i}}{\partial x}\right)+\frac{\partial}{\partial y}\left(\rho Y_{i} v-\rho D_{i} \frac{\partial Y_{i}}{\partial y}\right)=w_{i} \\
& \frac{\partial}{\partial t}(\rho)+\frac{\partial}{\partial x}(\rho u)+\frac{\partial}{\partial y}(\rho v)=0 \\
& \frac{\partial}{\partial t}(\rho u)+\frac{\partial}{\partial x}\left\{\rho u^{2}+p-\mu\left(\frac{4}{3} \frac{\partial u}{\partial x}-\frac{2}{3} \frac{\partial v}{\partial y}\right)\right\}+\frac{\partial}{\partial y}\left\{\rho u v-\mu\left(\frac{\partial v}{\partial x}+\frac{\partial u}{\partial y}\right)\right\}=0 \\
& \frac{\partial}{\partial t}(\rho v)+\frac{\partial}{\partial x}\left\{\rho u v-\mu\left(\frac{\partial v}{\partial x}+\frac{\partial u}{\partial y}\right)\right\}+\frac{\partial}{\partial y}\left\{\rho v^{2}+p-\mu\left(\frac{4}{3} \frac{\partial v}{\partial y}-\frac{2}{3} \frac{\partial u}{\partial x}\right)\right\}=0 \\
& \left.\left.\frac{\partial}{\partial t}\right)+\frac{\partial}{\partial x}\left\{\left(\rho e_{\mathrm{t}}+p\right) u-\kappa \frac{\partial T}{\partial x}-\sum\left(h_{i} \rho D_{i} \frac{\partial Y_{i}}{\partial x}\right)\right\}+\frac{\partial}{\partial y}\left\{\left(\rho e_{\mathrm{t}}+p\right) v-\kappa \frac{\partial T}{\partial y}-\sum_{i}\left(h_{i} \rho D_{i} \frac{\partial Y_{i}}{\partial y}\right)\right\}=H\left(T-T_{\mathrm{u}}\right)\right\}
\end{aligned}
$$

where $p$ is the pressure, $C_{i}$ is the molar concentration of $i$-th species, $T$ is the temperature, $\rho$ is the density, $Y_{i}$ is the mass fraction of $i$-th species, $u$ and $v$ are the velocities in the $x$ - and $y$-directions, $e_{\mathrm{t}}$ is the stored energy, $w_{i}$ is the reaction rate of $i$-th species, $R$ is the gas constant, $D_{i}$ is the diffusion coefficient of $i$-th species, $\mu$ is the dynamic viscosity, $\kappa$ is the thermal conductivity, $h_{i}$ is the enthalpy of $i$-th species, $H$ is the heat-loss parameter, and $T_{\mathrm{u}}$ is the temperature of the unburned gas. 
Kadowaki, Aung, Furuyama, Kawata, Katsumi and Kobayashi,

Journal of Thermal Science and Technology, Vol.16, No.2 (2021)

\section{Calculation procedures}

In numerical calculations, we adopted hydrogen-air lean premixed flames with the equivalence ratio of 0.5 and with the unburned-gas temperature of $298 \mathrm{~K}$. The pressure was set to $1 \mathrm{~atm}\left(1.013 \times 10^{5} \mathrm{~Pa}\right), 3 \mathrm{~atm}$ and 5 atm. The calculated burning velocities of planar flames $S_{\mathrm{u}}$ at $p=1,3$ and 5 atm under the adiabatic conditions were $0.50,0.36$ and $0.28 \mathrm{~m} / \mathrm{s}$, respectively. These burning velocities are consistent with the previous report (Dayma et al., 2014). Under the non-adiabatic conditions, a planar flame is extinguished at $H>H_{\max }$. The values of $H_{\max }$ were obtained by the calculations of planar flames with heat loss. The burning velocities of planar flames with heat loss of $H_{\max }$ at $p=1,3$ and 5 atm were $0.31,0.21$ and $0.18 \mathrm{~m} / \mathrm{s}$, respectively. The ratio of burning velocities under the non-adiabatic and adiabatic conditions is almost equal to the results of previous investigation (Joulin and Clavin, 1979).

Initial conditions for disturbed flames were provided with the solutions of stationary planar flames. On a planar flame, we superimposed a sinusoidal disturbance to calculate the unstable motion of a disturbed flame. The initial amplitude of a disturbance was set $A_{0}=0.02 \mathrm{~mm}$ for dispersion relations and $A_{0}=0.2 \mathrm{~mm}$ for cellular flames.

Boundary conditions in the $x$-direction were free-flow conditions, and the one-sided difference approximation with second-order accuracy was appropriated. The inlet-flow velocity was set equal to the burning velocity of a planar flame. Spatially periodic conditions were used in the $y$-direction.

Calculation domain $\left(L_{\mathrm{x}} \times L_{\mathrm{y}}\right)$ was $50 \mathrm{~mm}$ in the $x$-direction, and one to twelve times the wavelength $\lambda$ of a disturbance in the $y$-direction, which was resolved by $3881 \times 33$ spaced grid at $L_{y}=\lambda$ and $3881 \times 385$ spaced grid at $L_{y}=12 \lambda$. The minimum grid size in the $x$-direction was set to $0.01 \mathrm{~mm}$, and the used grid was fine enough to prevent numerical errors from contaminating the solutions. The time-step interval was $5 \mathrm{~ns}$.

\section{Dispersion relations}

To investigate the intrinsic instability of premixed flames, a sinusoidal disturbance with sufficiently small amplitude was superimposed on a planar flame. The amplitude $A$ grew exponentially with time, $A \sim \exp (\omega t)$, where $\omega$ is called the growth rate. The growth rate is defined as

$$
\omega=\frac{d}{d t} \ln \left(A / A_{0}\right)
$$

The behavior of the disturbed flame front, i.e. growing exponentially with time, was consistent with the prediction of linear analyses, and was observed only for sufficiently small amplitude. When the superimposed disturbance grew to some degree, the growth rate was gradually lowered. This was due to the nonlinearity brought about by finite amplitude.

The growth rate depends on the wavelength, i.e. on the wave number $k$, and the relation between the growth rate and wave number is called the dispersion relation. Figure 1 shows the dispersion relations at $p=1,3$ and 5 atm under the adiabatic conditions. As the pressure becomes higher, the maximum growth rate increases and the unstable range widens. This is due mainly to the decrease of the flame thickness induced by high pressure. The flame thickness is estimated by the unburned-gas temperature, burned-gas temperature and the maximum gradient of temperature; the estimated values at 1,3 and 5 atm are $0.50,0.19$ and $0.14 \mathrm{~mm}$, respectively. The dispersion relation yields the wave number corresponding to the maximum growth rate, i.e. the linearly most unstable wave number, and we refer to as the critical wave number $k_{\mathrm{c}}$. The higher the pressure, the larger the critical wave number. Thus, the critical wavelength $\lambda_{\mathrm{c}}\left(=2 \pi / k_{\mathrm{c}}\right)$ shortens as the pressure becomes higher. In small wave number region, moreover, we find that the growth rate decreases slightly as the pressure becomes higher. This is because of the decrease of the burning velocity of a planar flame.

The dispersion relations under the non-adiabatic conditions are shown in Fig. 2, where the values of $H_{\max }$ at $p=1,3$ and 5 atm are $1.35 \times 10^{5}, 5.30 \times 10^{5}$ and $8.55 \times 10^{5} \mathrm{~W} / \mathrm{m}^{3} \mathrm{~K}$, respectively. Compared with the adiabatic conditions, the growth rate is small and the unstable range is narrow. This is because that the burning velocity of a planar flame decreases owing to heat loss. In addition, we have smaller critical wave numbers, i. e. longer critical wavelengths, under the non-adiabatic conditions. 


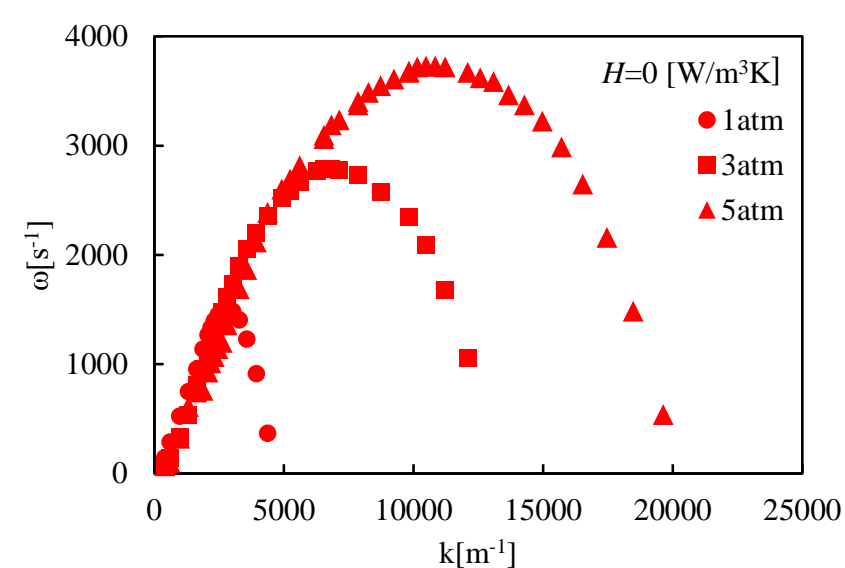

Fig. 1. Dispersion relations at $p=1,3$ and $5 \mathrm{~atm}$, under the adiabatic conditions.

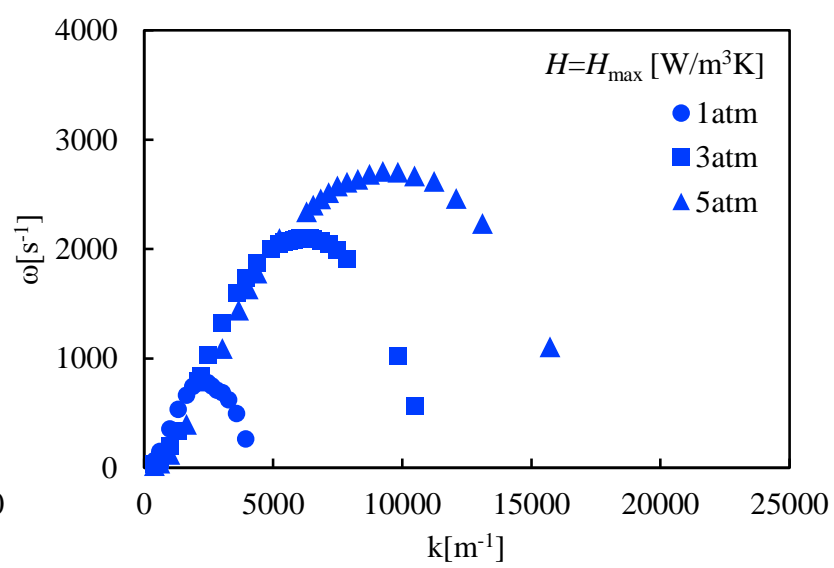

Fig. 2. Dispersion relations at $p=1,3$ and $5 \mathrm{~atm}$, under the non-adiabatic conditions.

\section{Cellular flames}

To investigate the characteristics of cellular flames caused by intrinsic instability, we superimposed a finite disturbance with the critical wavelength on a planar flame. Although disturbances with smaller amplitudes were superimposed, we obtained the same results on the unstable motion of cellular flames. One cell forms at small $L_{y}$, and multiple cells appear at sufficiently large $L_{y}$.

Figures 3 and 4 show the temperature distributions of cellular flames and concentration distributions of species at $L_{y}$ $=\lambda_{\mathrm{c}}$ under the adiabatic and non-adiabatic conditions, respectively, depending on the pressure. Unburned gas flows in from the left side, and burned gas flows out to the right side. In all cases, the superimposed disturbances evolve, and then cellular flames form, which is due to hydrodynamic and diffusive-thermal instabilities. As the pressure becomes higher, the cellular-flame shape becomes sharper, because the flame thickness decreases. In addition, we find slightly larger cell depth under the non-adiabatic conditions. This is because that the heat loss promotes the instability of premixed flames with low Lewis numbers. The pressure dependence of Lewis numbers is small enough in this investigation. In downstream of convex fronts toward to the unburned gas, the burned-gas temperature is higher than the temperature of a planar flame, which is because of the diffusive-thermal effects (Clavin, 1985). This overshoot of temperature is one of the significant factors of unstable motion of cellular-flame fronts. In the concentration distributions of $\mathrm{H}$ and $\mathrm{OH}$, lower concentrations are observed under the non-adiabatic conditions, which is induced by lower flame temperature. As the pressure becomes higher, the concentration of $\mathrm{H}$ increases slightly, even though the concentration of $\mathrm{OH}$ increases strongly. This is because that the dependence of $\mathrm{H}$ concentration on pressure is weak.

The temperature distributions of cellular flames at $p=5 \mathrm{~atm}$ and $L_{\mathrm{y}}=12 \lambda_{\mathrm{c}}$ under the adiabatic and non-adiabatic conditions, depending on the pressure, are shown in Figs. 5 and 6, respectively. The cellular-front shape is drastically changed with time, and several cells are observed. The combination of small cells and division of large cells also appear. The unstable motion of cellular-flame fronts becomes stronger with an increase in pressure, and the flame shape becomes more complicated. This is because diffusive-thermal instability becomes stronger owing to the decrease of the flame thickness induced by increasing pressure, and then the change of local burning velocity caused by diffusive-thermal effects at convex and concave fronts becomes greater. In addition, the motion of cellular-flame fronts under the nonadiabatic conditions is more unstable, even though the growth rate is smaller. This is because of the promotion of diffusive-thermal instability due to heat loss (Joulin and Clavin, 1979; Chao, 2001; Bechtold et al., 2005; Kadowaki, 2005). 
$\underline{1 \mathrm{~atm}}$

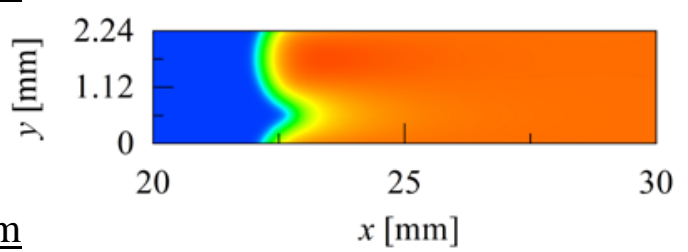

$\underline{\text { 3atm }}$

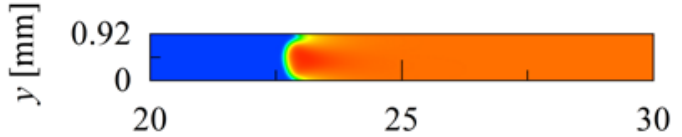

$\underline{5 a t m}$

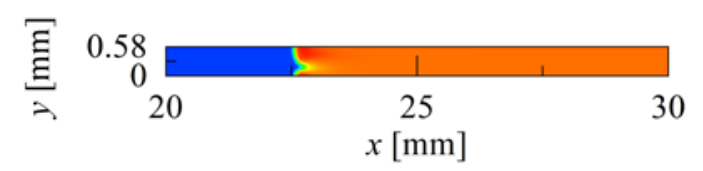

$\underline{1 \mathrm{~atm}}$

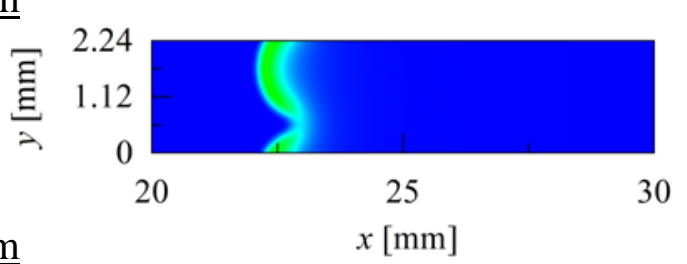

$\underline{3 a t m}$
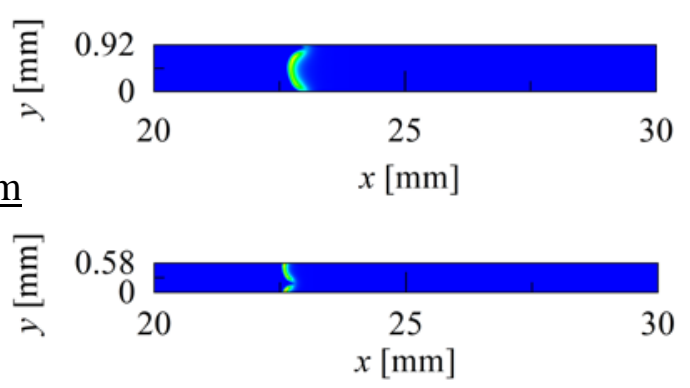

$\underline{1 \mathrm{~atm}}$

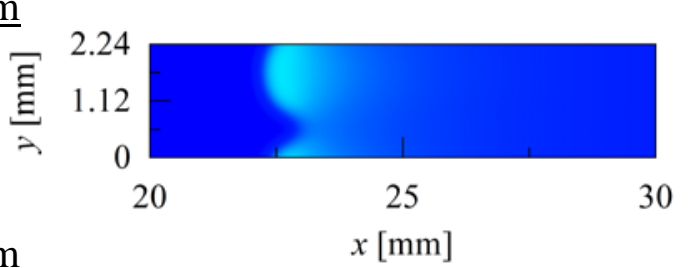

$\underline{3 a t m}$

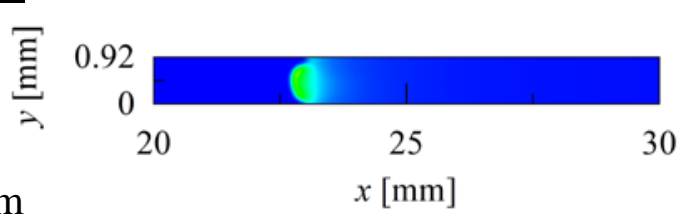

5atm

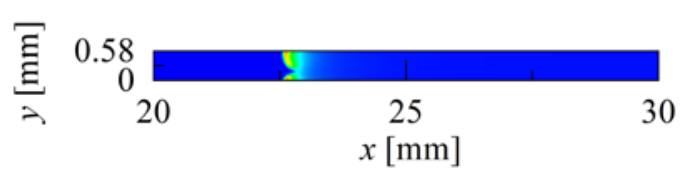

Fig. 3. Temperature distributions of cellular flames and concentration distributions of species at $L_{\mathrm{y}}=\lambda_{\mathrm{c}}$ under the adiabatic conditions, depending on the pressure.
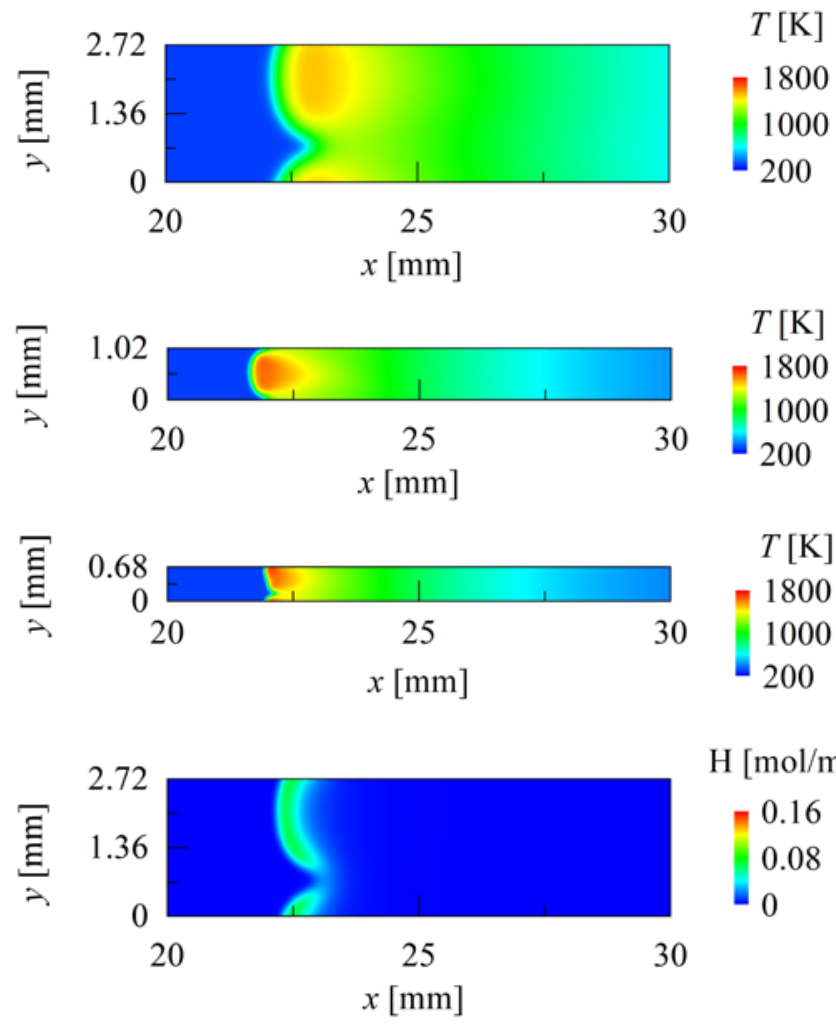

$\mathrm{H}\left[\mathrm{mol} / \mathrm{m}^{3}\right]$

0.16
0.08
0
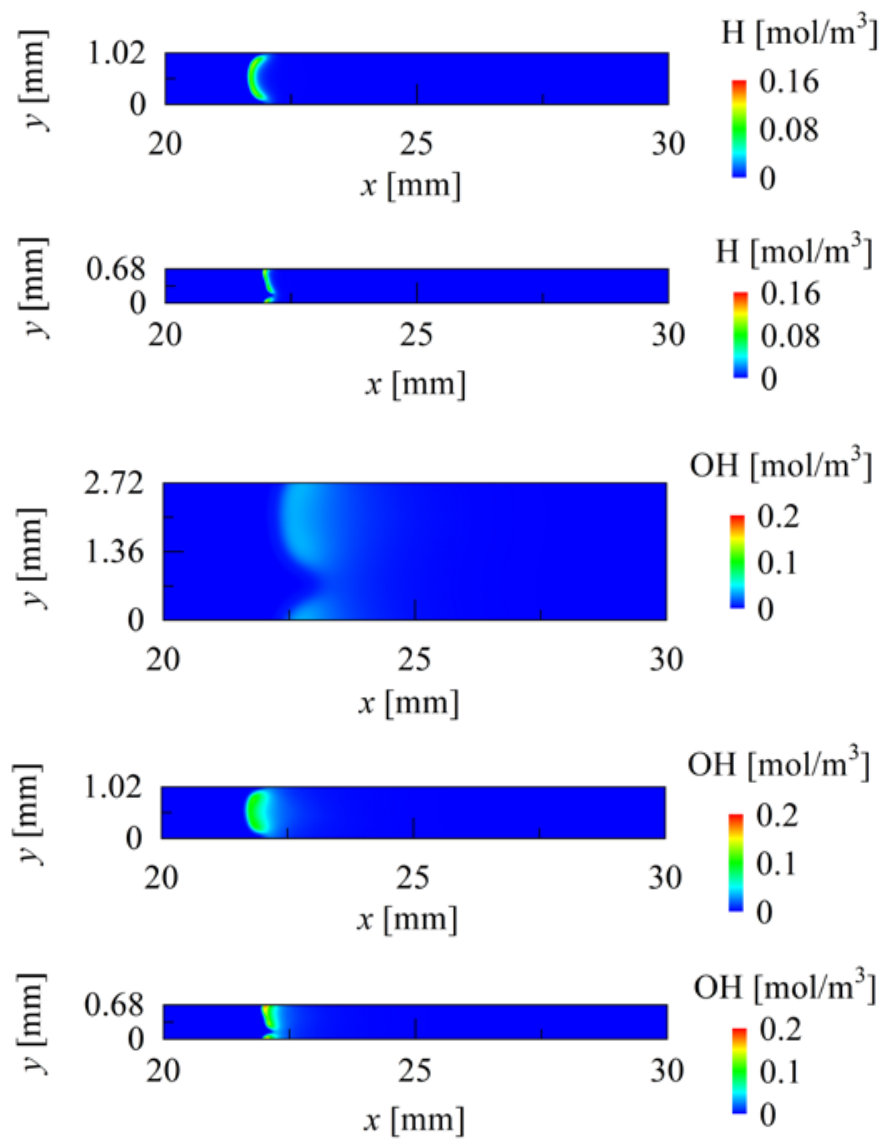

Fig. 4. Temperature distributions of cellular flames and concentration distributions of species at $L_{\mathrm{y}}=\lambda_{\mathrm{c}}$ under the non-adiabatic conditions, depending on the pressure. 
Kadowaki, Aung, Furuyama, Kawata, Katsumi and Kobayashi,

Journal of Thermal Science and Technology, Vol.16, No.2 (2021)

$\underline{1 \mathrm{~atm}}$

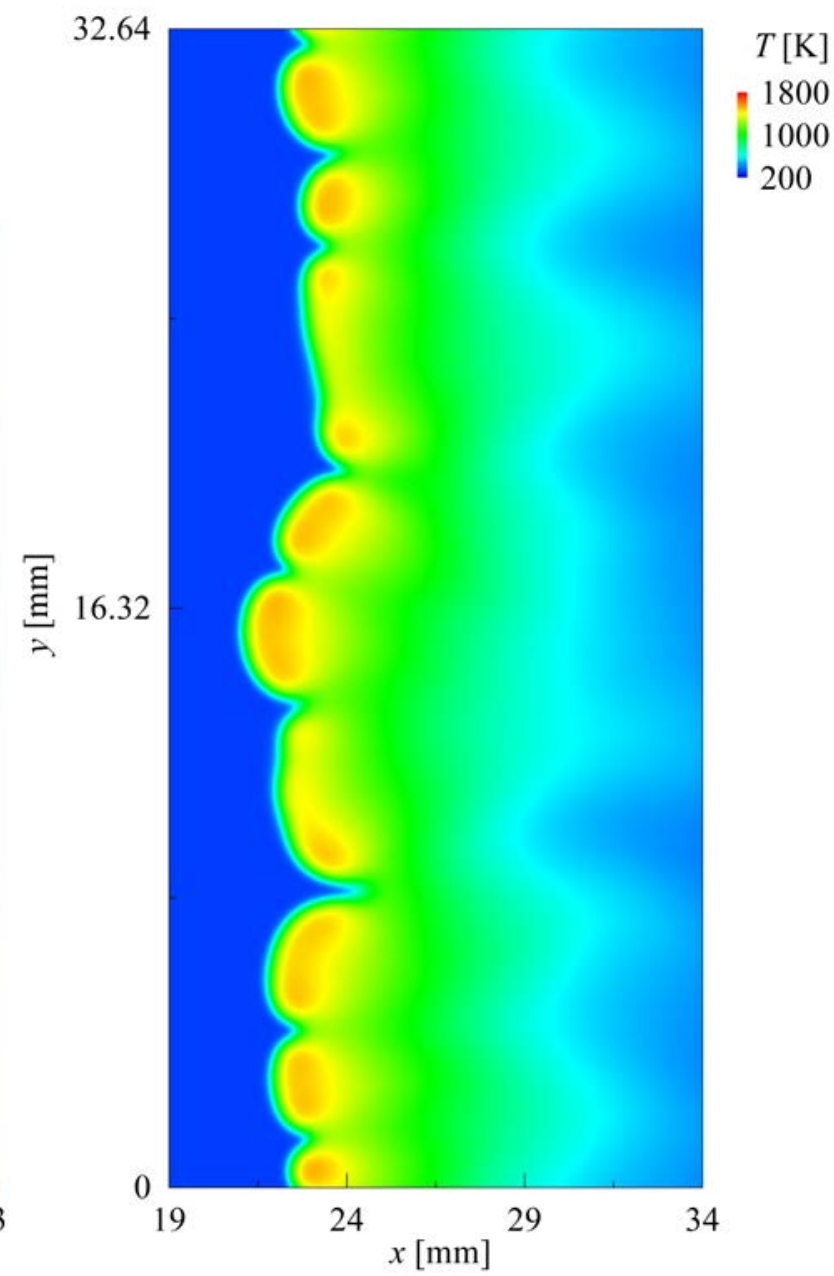

3atm
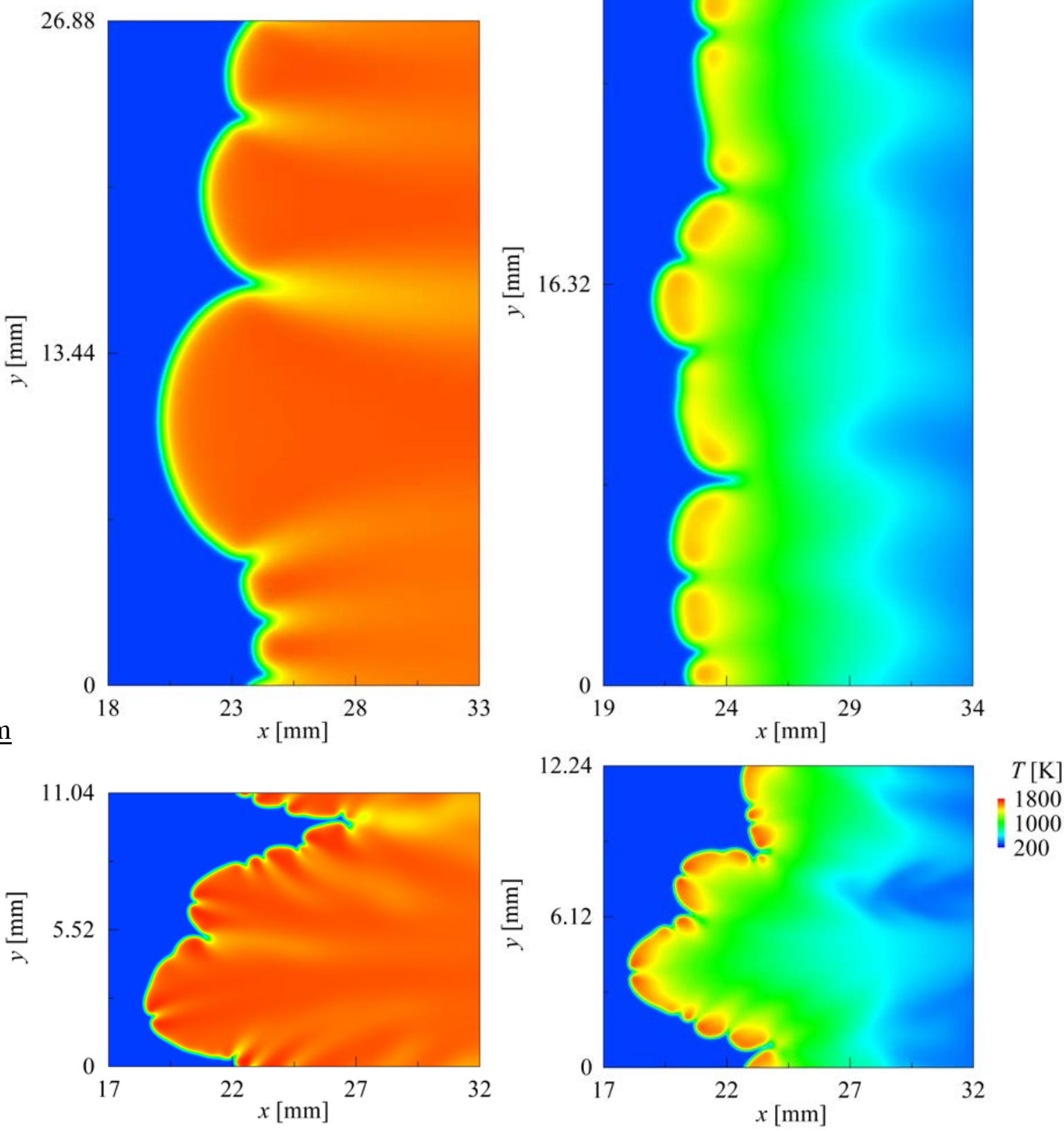

$\underline{5 a t m}$
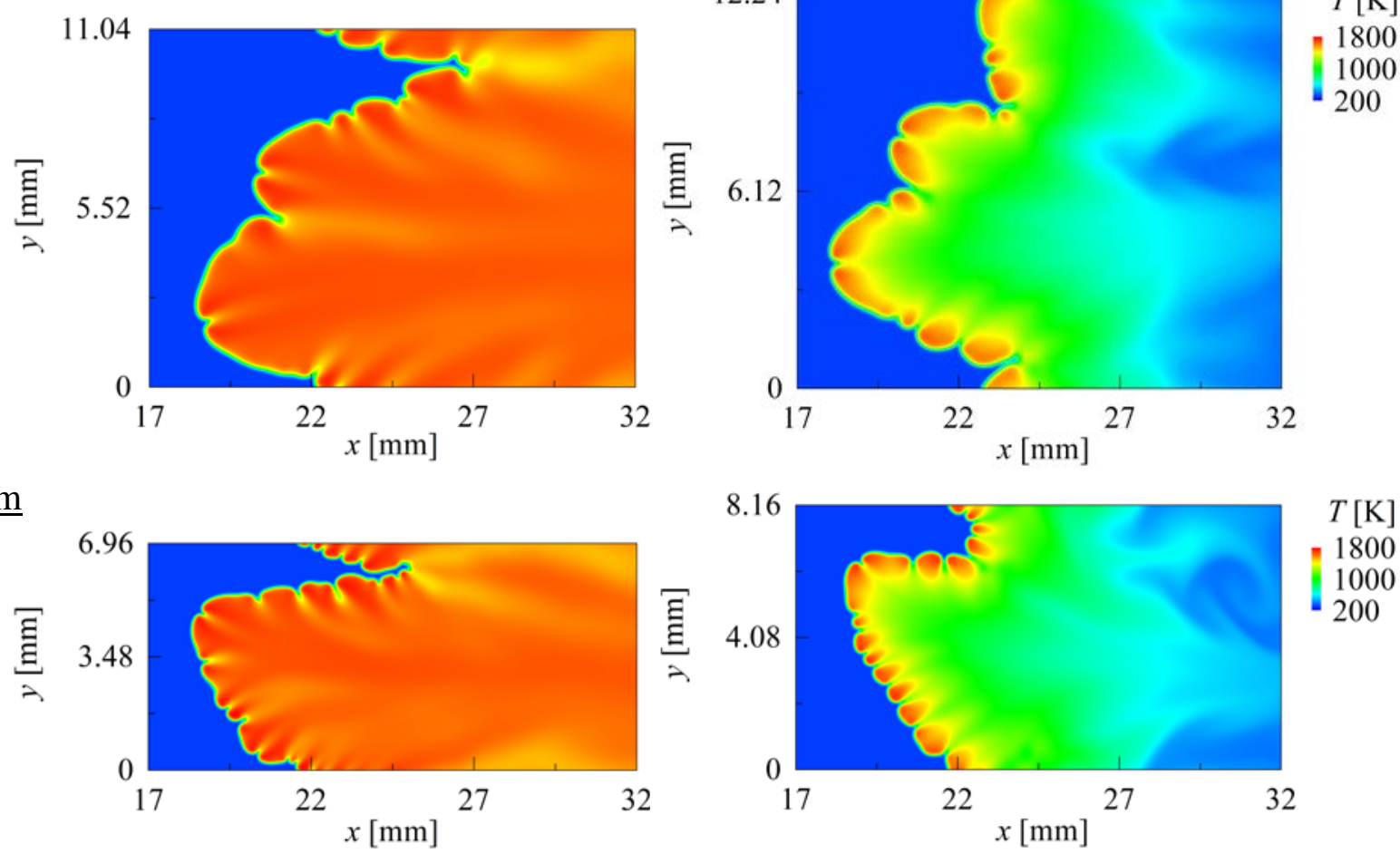

Fig. 5. Temperature distributions of cellular flames at $L_{\mathrm{y}}=12 \lambda_{\mathrm{c}}$ under the adiabatic conditions, depending on the pressure.

Fig. 6. Temperature distributions of cellular flames at $L_{\mathrm{y}}=12 \lambda_{\mathrm{c}}$ under the non-adiabatic conditions, depending on the pressure. 

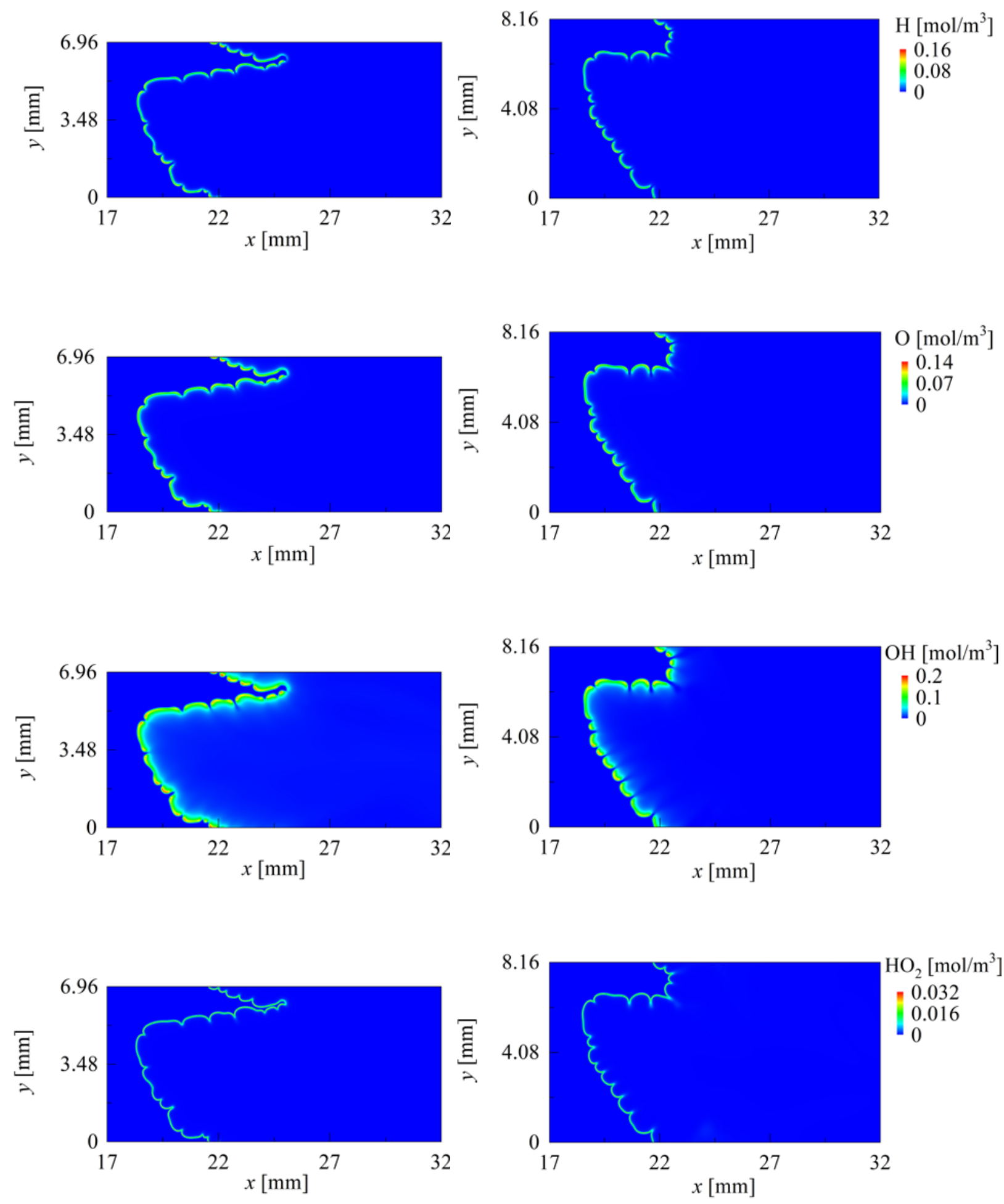

Fig. 7. Concentration distribution of species at $p=5$ atm and $L_{\mathrm{y}}=12 \lambda_{\mathrm{c}}$ under the adiabatic conditions.

Fig. 8. Concentration distribution of species at $p=5$ atm and $L_{\mathrm{y}}=12 \lambda_{\mathrm{c}}$ under the non-adiabatic conditions. 
The concentration distributions of species at $p=5 \mathrm{~atm}$ and $L_{\mathrm{y}}=12 \lambda_{\mathrm{c}}$ under the adiabatic and non-adiabatic conditions are shown in Figs. 7 and 8, respectively. In downstream of convex (concave) fronts, the concentrations of species are high (low) compared with the planar flames. This is induced by high (low) temperature at convex (concave) fronts due to the diffusive-thermal effects (Law, 1988).

Cellular flames have larger surface area, so that the burning velocity of a cellular flame is larger than that of a planar flame. Since the inlet-flow velocity is set equal to $S_{\mathrm{u}}$, a cellular flame moves upstream. The moving velocity $\Delta S$ corresponds to the increase in burning velocity. Thus, the burning velocity of a cellular flame $S_{\mathrm{cf}}$ is obtained by $S_{\mathrm{cf}}=S_{\mathrm{u}}$ $+\Delta S$. Figures 9 and 10 show the burning velocities of cellular flames normalized by those of planar flames at $p=1,3$ and $5 \mathrm{~atm}$ under the adiabatic and non-adiabatic conditions, respectively, depending on $L_{\mathrm{y}} / \lambda_{\mathrm{c}}$. In both figures, we show the relation between $S_{\mathrm{c}} / S_{\mathrm{u}}$ and $L_{\mathrm{y}} / \lambda_{\mathrm{c}}$ to elucidate the effects of space size on cellular instability essentially, because the critical wavelength, i.e. the typical length of intrinsic instability, depends strongly on the pressure. Adopting the normalized space size $L_{y} / \lambda_{c}$, we can clarify the space-size effects on the increase of burning velocity caused by intrinsic instability. As the pressure becomes higher, the normalized burning velocity of a cellular flame increases. In addition, the normalized burning velocity of a cellular flame increases monotonically as the normalized space size becomes larger. These results denote that the pressure and long-wavelength components of disturbances have a great influence on the unstable motion of cellular flames.

When the heat loss is taken into account, the unstable motion of cellular-flame fronts becomes stronger, and the burning velocity of a cellular flame normalized by that of a planar flame increases. This is because that the instability level becomes higher owing to the enlargement of Zeldovich numbers. In general, the Zeldovich number $\beta$ is defined as $\beta=E\left(T_{\mathrm{b}}-T_{\mathrm{u}}\right) / T_{\mathrm{b}}{ }^{2}$ (Niioka, 2004), where $E$ is the activation energy and $T_{\mathrm{b}}$ is the burned-gas temperature. Owing to the heat loss, the burned temperature decreases, and then the apparent Zeldovich number enlarges. Thus, the unstable motion becomes stronger under the non-adiabatic conditions in hydrogen-air lean premixed flames.

Finally, we estimate the fractal dimension of cellular-flame fronts. Using the concentration of $\mathrm{HO}_{2}$ of cellular flames shown in Figs.7 and 8. When we adopt the definition that the location of flame front is the position at the maximum gradient of temperature, the estimated fractal dimension is almost the same. We obtain the fractal dimension through the box-counting method (Miyauchi et al., 1994; Mukaiyama et al., 2013). The fractal dimension is obtained through the relation between the number of circles $N_{\mathrm{r}}$ and the radius of circles $r$ [mm] for cellular flames (see Fig. 11). The fractal dimension for two-dimensional flow $d$ is obtained by the following relation:

$$
d=-\frac{\log \left(N_{r}\right)}{\log (r)}
$$

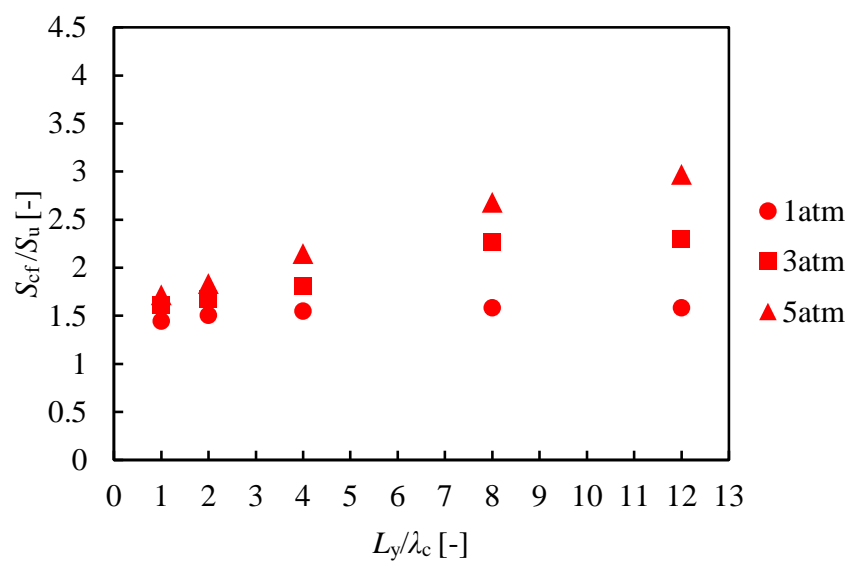

Fig. 9. Normalized burning velocities of cellular flames at $p=1,3$ and 5 atm under the adiabatic conditions, depending on $L_{\mathrm{y}} / \lambda_{\mathrm{c}}$.

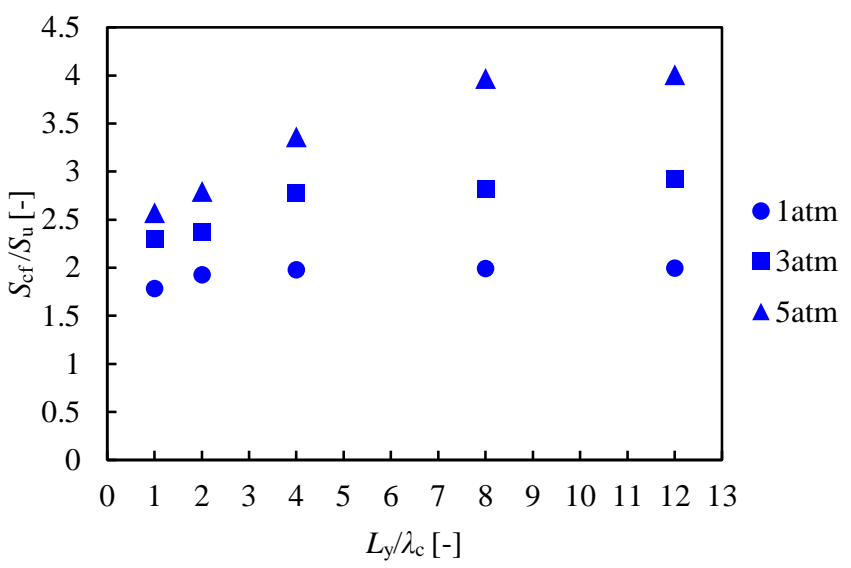

Fig. 10. Normalized burning velocities of cellular flames at $p=1,3$ and 5 atm under the non-adiabatic conditions, depending on $L_{\mathrm{y}} / \lambda_{\mathrm{c}}$. 


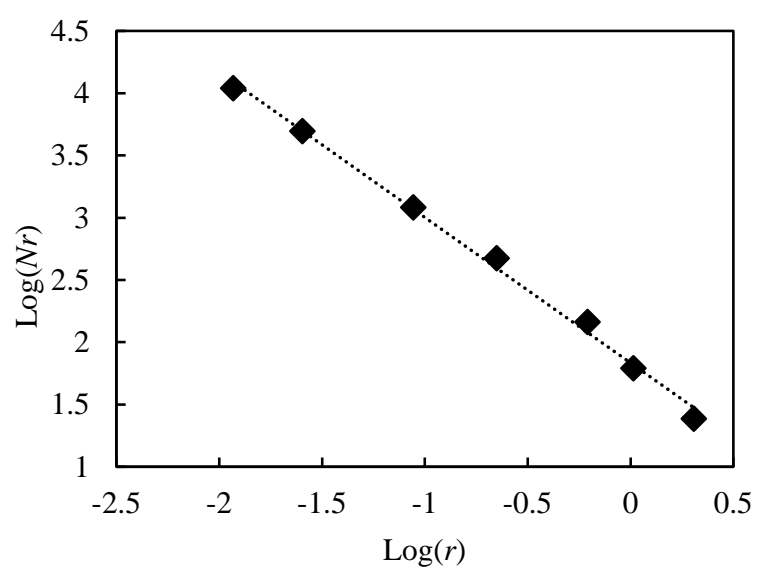

Fig. 11. Relations between $N_{r}$ and $r$ in cellular flames at $p=5$ atm and $L_{\mathrm{y}}=12 \lambda_{\mathrm{c}}$ under the non-adiabatic

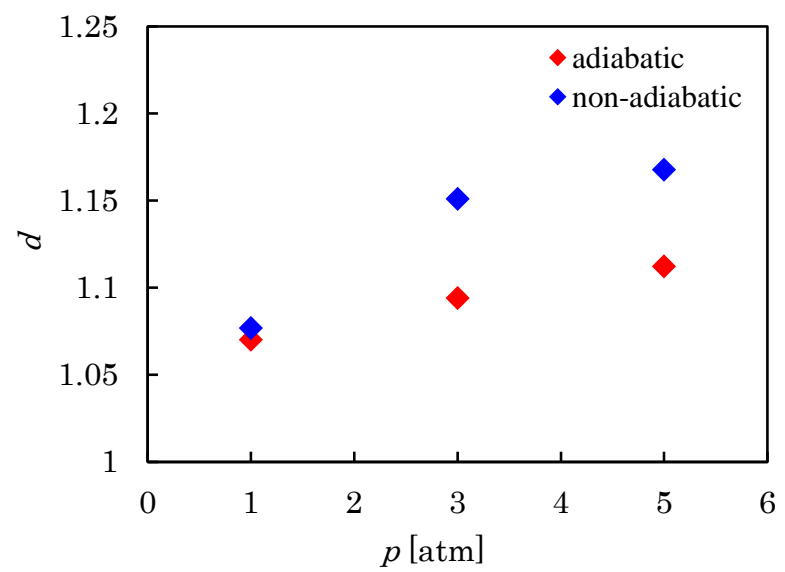

Fig. 12. Fractal dimensions of flame fronts at $L_{\mathrm{y}}=12 \lambda_{\mathrm{c}}$ under the adiabatic and non-adiabatic conditions, depending on the pressure.

From the gradients of lines shown in Fig. 11, we get the fractal dimension. In addition, it is confirmed that the fractal dimension is almost constant at the space size larger than $8 \lambda_{\mathrm{c}}$. As the pressure becomes higher, the fractal dimension increases (see Fig. 12). The results indicate that the flame front becomes increasingly complex as the pressure becomes higher. In addition, the fractal dimension under the non-adiabatic conditions is larger. This denotes that the flame shape becomes more complicated, because the heat loss promotes diffusive-thermal instability.

\section{Conclusions}

We have calculated two-dimensional unsteady reactive flow, based on the detailed chemical reaction model, to investigate the effects of pressure and heat loss on the unstable motion of cellular-flame fronts. Through numerical calculations, we have obtained the following results.

In the dispersion relations, the maximum growth rate increases and the unstable range widens as the pressure becomes higher. These are due mainly to the decrease of flame thickness. The growth rate decreases and the unstable range narrows as the heat loss becomes larger, which are due mainly to the decrease of burning velocity. In the investigation on the characteristics of cellular flames, a disturbance with the critical wavelength is superimposed. The superimposed disturbance develops owing to intrinsic instability, and then the cellular shape of flame fronts appears. The normalized burning velocity of a cellular flame increases as the pressure becomes higher and the heat loss becomes larger, indicating that the pressure and heat loss affect strongly the unstable motion of cellular-flame fronts. The burning velocity of a cellular flame increases monotonically with an increase in the space size, which is attributed to long-wavelength components of disturbances. Moreover, we estimate the fractal dimension to clarify the complexity of flame fronts. As the pressure and heat loss increase, the fractal dimension becomes larger, which denotes that the flame shape becomes more complicated.

In this numerical investigation, we treat the two-dimensional reactive flow. In the near future, we will try threedimensional calculations to compare the flame characteristics between two- and three-dimensional fields. Moreover, we would like to perform the experiments of hydrogen-air premixtures to reveal details of the pressure effects on the characteristics of cellular flames. 
Kadowaki, Aung, Furuyama, Kawata, Katsumi and Kobayashi,

Journal of Thermal Science and Technology, Vol.16, No.2 (2021)

\section{References}

Battista, F., Troiani, G. and Picano, F., Fractal scaling of turbulent premixed flame fronts: application to LES, International Journal of Heat and Fluid Flow, Vol. 51 (2015), pp. 78-87.

Bechtold, J. K., Cui, C. and Matalon, M., The role of radiative losses in self-extinguishing and self-wrinkling flames, Proceedings of the Combustion Institute, Vol. 30 (2005), pp. 177-184.

Bradley, D., Lawes, M., Liu, K., Verhelst, S. and Woolley, R., Laminar burning velocities of lean hydrogen-air mixtures at pressures up to 1.0 MPa, Combustion and Flame, Vol. 149 (2007), pp. 162-172.

Chao, B. H., Instability of burner-stabilized flames with volumetric heat loss, Combustion and Flame, Vol. 126 (2001), pp. 1476-1488.

Clavin, P., Dynamic behavior of premixed flame fronts in laminar and turbulent flows, Progress in Energy and Combustion Science, Vol. 11 (1985), pp. 1-59.

Coffee, T. P. and Heimerl, J. M., Transport algorithms for premixed, laminar steady-state flames, Combustion and Flame, Vol. 43 (1981), pp. 273-289.

Dayma, G., Halter, F. and Dagaut, P., New insights into the peculiar behavior of laminar burning velocities of hydrogenair flames according to pressure and equivalence ratio, Combustion and Flame, Vol. 161 (2014), pp. 2235-2241.

Frouzakis, C. E., Fogla, N., Tomboulides, A. G., Altantzis, C. and Matalon, M., Numerical study of unstable hydrogen/air flames: shape and propagation speed, Proceedings of the Combustion Institute, Vol. 35 (2015), pp. 1087-1095.

Gavrikov, A. I., Golub, V. V., Mikushkin, A. Y., Petukhov, V. A. and Volodin, V. V., Lean hydrogen-air premixed flame with heat loss, International Journal of Hydrogen Energy, Vol. 44 (2019), pp. 20462-20469.

Gubernov, V. V., Bykov, V. and Maas, U., Hydrogen/air burner-stabilized flames at elevated pressures, Combustion and Flame, Vol. 185 (2017), pp. 44-52.

Hertzberg, M., Selective diffusional demixing: occurrence and size of cellular flames, Progress in Energy and Combustion Science, Vol. 15 (1989), pp. 203-239.

Hirschfelder, I. O., Curtiss, C. F. and Bird, R. B., Molecular Theory of Gases and Liquids (1954), p. 8, John Wiley \& Sons, New York.

Hu, E., Huang, Z., He, J., Zheng, J. and Miao, H., Measurements of laminar burning velocities and onset of cellular instabilities of methane-hydrogen-air flames at elevated pressures and temperatures, International Journal of Hydrogen Energy, Vol. 34 (2009a), pp. 5574-5584.

Hu, E., Huang, Z., He, J. and Miao, H., Experimental and numerical study on laminar burning velocities and flame instabilities of hydrogen-air mixtures at elevated pressures and temperatures, International Journal of Hydrogen Energy, Vol. 34 (2009b), pp. 8741-8755.

Ichikawa, Y., Otawara, H., Kobayashi, H., Ogami, Y., Kudo, T., Okuyama, M. and Kadowaki, S., Flame structure and radiation characteristics of $\mathrm{CO} / \mathrm{H}_{2} / \mathrm{CO}_{2} /$ air turbulent premixed flames at high pressure, Proceedings of the Combustion Institute, Vol. 33 (2011), pp. 1543-1550.

Imteyaz, B. A., Nemitallah, M. A., Abdelhafez, A. A., Habib, M. A., Combustion behavior and stability map of hydrogenenriched oxy-methane premixed flames in a model gas turbine combustor, International Journal of Hydrogen Energy, Vol. 43 (2018), pp. 16652-16666.

Joulin, G. and Clavin, P., Linear stability analysis of nonadiabatic flames: diffusional-thermal model, Combustion and Flame, Vol. 35 (1979), pp. 139-153.

Kadowaki, S., The effects of heat loss on the burning velocity of cellular flames generated by hydrodynamic and diffusive-thermal instabilities, Combustion and Flame, Vol. 143 (2005), pp. 174-182.

Kadowaki, S. and Hasegawa, T., Numerical simulation of dynamics of premixed flames: flame instability and vortexflame interaction, Progress in Energy and Combustion Science, Vol. 31 (2005), pp. 193-241.

Kadowaki, S., Uchiyama, T., Katsumi, T., Thwe Thwe Aung and Kobayashi, H., Unstable behavior of hydrogen-air lean premixed flames (Effects of unburned-gas temperature, heat loss and scale), Transactions of the JSME (in Japanese), Vol.86, No.883, (2020), DOI: 10.1299/ transjsme.19-00401.

Kadowaki, S., Yanagioka, T., Yamazaki, W. and Kobayashi, H., The intrinsic instability of three-dimensional premixed flames under the low- and high-temperature conditions: effects of unburned-gas temperature on hydrodynamic and diffusive-thermal instabilities, Combustion Science and Technology, Vol. 187 (2015), pp. 1167-1181. 
Katsumi, T., Kobayashi, H., Aida, T., Aiba, K. and Kadowaki, S., Outward propagation velocity and acceleration characteristics in hydrogen-air deflagration, International Journal of Hydrogen Energy, Vol. 42 (2017), pp. 73607365.

Kim, W. K., Mogi, T. and Dobashi, R., Fundamental study on accidental explosion behavior of hydrogen-air mixtures in an open space, International Journal of Hydrogen Energy, Vol. 38 (2013), pp. 8024-8029.

Law, C. K., Dynamics of stretched flames, Proceedings of the Combustion Institute, Vol. 22 (1988), pp. $1381-1402$.

Mitani, T. and Williams, F. A., Studies of cellular flames in hydrogen-oxygen-nitrogen mixtures, Combustion and Flame, Vol. 39 (1980), pp. 169-190.

Miyauchi, T., Tanahashi, M. and Gao, F., Fractal characteristics of turbulent diffusion flames, Combustion Science and Technology, Vol. 96 (1994), pp. 135-154.

Mukaiyama, K., Shibayama, S. and Kuwana, K., Fractal structures of hydrodynamically unstable and diffusive-thermally unstable flames, Combustion and Flame, Vol. 160 (2013), pp. 2471-2475.

Nakahara, M., Abe, F., Tokunaga, K. and Ishihara, A., Effect of dilution gas on burning velocity of hydrogen-premixed mesoscale spherical laminar flames, Proceedings of the Combustion Institute, Vol. 35 (2015), pp. 639-646.

Niioka, T., Analysis of Combustion Phenomena (2004), p. 5, Tohoku University Press.

Okafor, E. C., Hayakawa, A., Nagano, Y. and Kitagawa, T., Effects of hydrogen concentration on premixed laminar flames of hydrogen-methane-air, International Journal of Hydrogen Energy, Vol. 39 (2014), pp. 2409-2417.

Park, J. and Lee, M. C., Combustion instability characteristics of $\mathrm{H}_{2} / \mathrm{CO} / \mathrm{CH}_{4}$ syngases and synthetic natural gases in a partially-premixed gas turbine combustor: Part I - Frequency and mode analysis, International Journal of Hydrogen Energy, Vol. 41 (2016a), pp. 7484-7493.

Park, J. and Lee, M. C., Combustion instability characteristics of $\mathrm{H}_{2} / \mathrm{CO} / \mathrm{CH}_{4}$ syngases and synthetic natural gases in a partially-premixed gas turbine combustor: Part II - Time lag analysis, International Journal of Hydrogen Energy, Vol. 41 (2016b), pp. 1304-1312.

Pizza, G., Mantzaras, J., Frouzakis, C. E., Tomboulides, A. G. and Boulouchos, K., Suppression of combustion instabilities of premixed hydrogen/air flames in microchannels using heterogeneous reactions, Proceedings of the Combustion Institute, Vol. 32 (2009), pp. 3051-3058.

Qin, X., Kobayashi, H. and Niioka, T., Laminar burning velocity of hydrogen-air premixed flames at elevated pressure, Experimental Thermal and Fluid Science, Vol. 21 (2000), pp. 58-63.

Sivashinsky, G. I., On the intrinsic dynamics of premixed flames, Philosophical Transactions of the Royal Society of London, Series A, Vol. 332 (1990), pp. 135-148.

Yanez, J., Kuznetsov, M. and Grune, J., Flame instability of lean hydrogen-air mixtures in a smooth open-ended vertical channel, Combustion and Flame, Vol. 162 (2015), pp. 2830-2839.

Yenerdag, B., Minamoto, Y., Naka, Y., Shimura, M. and Tanahashi, M., Flame propagation and heat transfer characteristics of a hydrogeneair premixed flame in a constant volume vessel, International Journal of Hydrogen Energy, Vol. 41 (2016), pp. 9679-9689.

Westbrook, C. K., Hydrogen oxidation kinetics in gaseous detonations, Combustion Science and Technology, Vol. 29 (1982), pp. 67-81.

Wilke, C. R., Diffusional properties of multicomponent gases, Chemical Engineering Progress, Vol. 46 (1950a), pp. 95104.

Wilke, C. R., A viscosity equation for gas mixtures, Journal of Chemical Physics, Vol. 18 (1950b), pp. 517-519.

Williams, F. A., Combustion Theory, Second Edition (1985), p. 349, Addison-Wesley, Reading, CA.

Xiao, J., Travis, J. R. and Kuznetsov, M., Numerical investigations of heat losses to confinement structures from hydrogen-air turbulent flames in ENACCEF facility, International Journal of Hydrogen Energy, Vol. 40 (2015), pp. 13106-13120. 